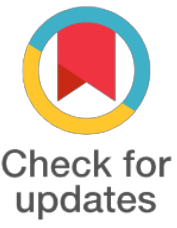

Check for updates

*For correspondence:

hindIII@yandex.ru

Competing interests: The authors declare that no competing interests exist.

Received: 2017-03-27

Accepted: 2017-06-28

Published: 2017-09-05

Copyright The Author(s) 2017. This article is published with open access by BioMedPress (BMP).

This article is distributed under the terms of the Creative Commons Attribution License (CC-BY 4.0) which permits any use, distribution, and reproduction in any medium, provided the original author(s) and the source are credited.

\section{Crosstalk between effector cells of adaptive immunity and mesenchymal stromal cells}

\author{
Aleksandra Gornostaeva, Gornostaeva Aleksandra, Andreeva Elena, Buravkova \\ Ludmila
}

Khoroshevskoe shosse, 76a Moscow 123007, Russia

\section{Abstract}

Multipotent mesenchymal stromal cells (MSCs) are a perspective tool for regenerative medicine due low immunogenicity and immunomodulation. The "feedback loop" exists in MSC/immune cells relationships, when "inflammatory" stimulation switches immunoregulaton by MSCs. Currently, the most studied effect of allogeneic MSCs on adaptive immunity cells, mainly on T lymphocytes. Studies of the interaction of MSCs and innate immunity cells are much less. "Reverse effects" (the effect of immune cells on MSCs) are virtually not investigated. Initiation of the inflammation occurs with activation of innate immunity cells, that "turns on" immunomodulatory properties. In this regard, the study of the interaction of MSCs and monocytes is particularly relevant. MSCs from human adipose tissue and CD14+monocytes (MNs) from peripheral blood of healthy volunteers were used. To stimulate monocytes conditioned medium (CM) after 72 hours of mixed lymphocyte reaction (MLR) was applied. This CM was enriched with IL-8, INF-gamma and TNF-a. Optimization of MN activation procedure was performed prior to experiments. CD14+MNs were incubated with different concentration of MLRCM for a different time. The activation and viability of MNs was evaluated every 24 hours. The overnight exposure of MNs to 3-day 50\% CM-MLR was found to be optimum regime. We studied the of $\mathrm{MSC} /$ monocyte interaction paying special attention to "feedback loop". In the presence of activated MNs, MSCs possessed unchanged viability (96\%), transmembrane mitochondria potential, ROS level and twice reduced lysosome activity. The cytokine profile in coculture medium was changed significantly. IL-6 and MCP-1 were increased vs monocultures of both cell types. IL-8 was similar to MN monoculture. TNF alpha, MIG, IL-10 were detected as tracers. Elevation of IL- 6 and MIG indicates on acquisition of anti-inflammatory phenotype by MSCs. After interaction with MSC, the share of CD69+ MNs (nonspecific marker of early activation) decreased, HLA-DR (MHC class II receptor) increased slightly. A threefold increase in both CD163+ MN's share and MFI was detected, whereas CD86 antigen was not expressed. The changes in the cytokine profile and the expression of surface markers described above are characteristic of the anti-inflammatory phenotype of monocytes. Thus, upon interaction MSC exhibited pronounced immunomodulatory properties and shifted the phenotype of monocytes towards the anti-inflammatory. These data indicate on the MSC potential to modulate early stages of inflammation, while retaining their functional state. 


\section{Keywords}

immunomodulation, MSC, immune cells, interaction

Funding

This work was supported in part by Programm of RAS Presidium "Integrative physiology", RFBR grant 17-04-00942, grant of the President of the Russian Federation SP-3502.2015.4

References 